\title{
OPEN Transcriptomic analysis of Rhodococcus opacus R7 grown on polyethylene by RNA-seq
}

\author{
Jessica Zampolli ${ }^{1}$, Alessandro Orro ${ }^{2}$, Andrea Manconi ${ }^{2}$, Diletta Ami ${ }^{1}$, Antonino Natalello ${ }^{1}$ \& \\ Patrizia Di Gennaro ${ }^{1 凶}$
}

Plastic waste management has become a global issue. Polyethylene (PE) is the most abundant synthetic plastic worldwide, and one of the most resistant to biodegradation. Indeed, few bacteria can degrade polyethylene. In this paper, the transcriptomic analysis unveiled for the first time Rhodococcus opacus R7 complex genetic system based on diverse oxidoreductases for polyethylene biodegradation. The RNA-seq allowed uncovering genes putatively involved in the first step of oxidation. In-depth investigations through preliminary bioinformatic analyses and enzymatic assays on the supernatant of R7 grown in the presence of PE confirmed the activation of genes encoding laccase-like enzymes. Moreover, the transcriptomic data allowed identifying candidate genes for the further steps of short aliphatic chain oxidation including alkB gene encoding an alkane monooxygenase, cyp450 gene encoding cytochrome $\mathrm{P} 450$ hydroxylase, and genes encoding membrane transporters. The PE biodegradative system was also validated by FTIR analysis on R7 cells grown on polyethylene.

Plastics are an extensive group of synthetic polymers of fossil origin (i.e., crude oil and gas) or renewable (i.e., sugar cane, starch, and vegetable oils), or even mineral base (salt). In the last two centuries, plastic played a revolutionary role for its versatile properties, including convenience, non-degradability, durability, and low cost replacing other natural materials for packaging, transportation, storage, and garbage ${ }^{1}$. In 2018 plastics production almost reached 62 million tonnes only in Europe, and packaging by far represents the largest end-use markets ${ }^{2}$.

The most used synthetic polymer is polyethylene (PE) for its chemical-physical and mechanical properties such as high hydrophobicity, chemical resistance, electrical isolation, high breaking strength, high stability against deterioration, and low production costs ${ }^{3}$. In general, the Global Plastic industry is growing at a rate of $4.8 \%$ annually, significantly impacting the growth of the polyethylene market whose value is expected to rise to 215 billion U.S. dollars by $2024^{4}$. Different types of PE can be distinguished based on their density, the degree of branching, and the availability of functional groups on the surface: Low-Density Polyethylene (LDPE), High-Density Polyethylene (HDPE), Linear Low-Density Polyethylene (LLDPE), and Cross-Linked Polyethylene (XLPE) ${ }^{5}$.

Although the properties of $\mathrm{PE}$ are favorable for its long lifetime and durability, the same characteristic can lead to long-term impacts and risks as a major source of pollution for the environment. Indeed, the main issue is represented by the management of the PE end of life and waste disposal that still need to be addressed ${ }^{6,7}$. PE has always been considered as a chemically and biologically inert polymer with a degradation yield (mechanical or biodegradation) that can occur to a certain extent. The European Directive (UNI EN 13432:2002, 2002) defines biodegradable if more than $90 \%$ of the initial compound can be transformed into biomass, water, and $\mathrm{CO}_{2}$ within 6 months. Rather, PE deterioration requires a prolonged period under environmental conditions and some research studies reported microbial PE-degradative capacity ${ }^{5,8,9}$. Moreover, Albertsson and coworkers ${ }^{10}$ suggested that a synergistic effect between photooxidative degradation and biodegradation can facilitate the PE degradation: UV light and/or oxidizing agents begin the degradation, and after carbonyl group formation, microorganisms degrade the shorter segments starting from the oxidized PE chains forming carbon dioxide and water as end products. Indeed, different reports showed bacterial biodegradation of PE but only after the polymer was treated with acid ${ }^{11}$ or subjected to photooxidation or high temperatures beforehand ${ }^{12-14}$.

$\mathrm{PE}$ mineralization is a complex process that comprises the first oxidation of the hydrocarbon chain generating shorter aliphatic fragments and a consecutive degradation of the generated short alkanes ${ }^{15}$. PE biodegradability

\footnotetext{
${ }^{1}$ Department of Biotechnology and Biosciences, University of Milano-Bicocca, Piazza della Scienza 2, 20126 Milan, Italy. ${ }^{2}$ Institute of Biomedical Technologies, National Research Council, CNR, via Fratelli Cervi 19, Segrate, 20133 Milan, Italy. ${ }^{\square}$ email: patrizia.digennaro@unimib.it
} 
is inverse to its molecular weight: higher is the molecular weight, $\mathrm{PE}$ is more resistant to biodegradation whereas the higher number of branched molecules of LDPE can improve biodegradation ${ }^{16}$.

Proteobacteria and Firmicutes are two of the most common phyla of plastic degraders and more recently Actinobacteria demonstrated to have an important role in PE-contaminated soils ${ }^{9}$. Up to now, only 20 different bacterial genera are known to degrade diverse types of PE, including Gram-negative and Gram-positives bacteria belonging to Pseudomonas, Ralstonia, Stenotrophomonas, Klebsiella, Acinetobacter, Staphylococcus, Streptococcus, Streptomyces, Micrococcus, Nocardia, Brevibacillus, and Bacillus genera ${ }^{1,5,17}$. Among the others, few bacteria belonging to Rhodococcus genus possess the ability to oxidize and degrade PE. Microorganisms belonging to this genus are usually characterized by a versatile metabolism vs different organic contaminants, metals, and drugs and a unique adaptation to fluctuating and harsh environmental circumstances ${ }^{14,15,18,19}$. Moreover, it was demonstrated that only Rhodococcus ruber strain C208 was able to mineralize PE with and without prior abiotic factors, and RNA-seq analysis showed the induction of the pathways related to alkane degradation (alkB genes) and $\beta$-oxidation of fatty acids.

The involvement of the alkane degradation system was also demonstrated for Pseudomonas aeruginosa E7 and other Pseudomonas strains ${ }^{20,21}$. The AlkB engaging is likely explicable with the presence in PE samples or the formation of short aliphatic fragments, which could be recognized as the natural substrates/inducers. However, the complex mechanism of PE degradation requires prime oxidation to generate shorter aliphatic molecules as the first step ${ }^{15}$. In this perspective, Santo and coworkers ${ }^{22}$ reported a laccase-like multi-copper oxidase (LMCO) isolated from $R$. ruber C208 that has been proved to be able, when overproduced, to induce a reduction of the PE molecular weight. Nonetheless, transcriptomic analysis of C208 after growth on PE did not show any upregulated laccases that are preferentially induced by lignin or aromatic compounds related to lignin or by metals, such as $\mathrm{Cu}^{2+}$.

In order to unravel the complex degradative system behind PE mineralization and identify the genetic determinants involved in this metabolism, a combination of genome-level techniques can help; thus, bacteria belonging to Rhodococcus genus can represent the perfect candidate for digging deeper.

In this paper, we report the transcriptomic analysis of Rhodococcus opacus strain R7 grown on PE to elucidate the biodegradative mechanism of polyethylene. RNA-seq allowed recovering genes putatively involved in the first step of oxidation. In-depth investigations through preliminary bioinformatic analyses and enzymatic assays on the supernatant of R7 grown in the presence of PE confirmed the activation of genes encoding laccaselike enzymes. Moreover, the transcriptomic data allowed identifying candidate genes for the further steps of short aliphatic chain oxidation including alkB gene encoding an alkane monooxygenase, cyp450 gene encoding cytochrome $\mathrm{P} 450$ hydroxylase, and genes encoding membrane transporters. In addition, the PE biodegradative system was also validated by FTIR analysis on R. opacus R7 cells grown on polyethylene.

\section{Material and methods}

Bacterial strain and growth conditions. R. opacus strain R7 (CIP identification number 107348, deposited to the Institute Pasteur Collection) ${ }^{23,24}$ isolated from PAH contaminated soil, was generally cultivated in M9 mineral medium ${ }^{25}$ supplemented with $20 \mathrm{mM}$ malate. Rapid screening growth assay was performed inoculating R. opacus R7 on M9 mineral medium agar plates supplemented with $1.2 \%$ polyethylene powder (Sigma-Aldrich 427772 , Italy) in $1 \%$ Tween 80 after vortexing for $1 \mathrm{~min}$ and then incubating at $30^{\circ} \mathrm{C}$ for $48 \mathrm{~h}$.

The growth assay was performed with a two-step inoculum: the R7 malate overnight culture was washed in mineral medium and inoculated in fresh M9 mineral medium supplemented with polyethylene powder (1.2\%) as only carbon and energy source. After $48 \mathrm{~h}$ of growth, the PE culture was renovated in fresh M9 mineral medium supplemented with $1.2 \% \mathrm{PE}$ powder. The inoculum was incubated at $30^{\circ} \mathrm{C}$ under shaking (120 rpm) up to $144 \mathrm{~h}$. The cultures were harvested at the late-exponential growth phase (optical density at $600 \mathrm{~nm}$ of $0.5 \pm 0.05$ ).

The R7 growth was evaluated also with the colony-forming unit counting method by serially diluting $0.1 \mathrm{~mL}$ of bacterial cultures in a solution of M9 mineral medium and plating on Luria-Bertani (LB) agar medium. Plates were incubated at $30^{\circ} \mathrm{C}$ for $48 \mathrm{~h}$. Data reported the mean of three biological replicates with standard deviation.

$\mathrm{R} 7$ culture in mineral medium M9 with $20 \mathrm{mM}$ malate grown overnight at $30^{\circ} \mathrm{C}(120 \mathrm{rpm})$ represented the reference condition. All conditions were performed in triplicates.

Illumina high-throughput transcriptome sequencing. RNA extraction. Total RNA was isolated from R. opacus $\mathrm{R} 7$ cells (up to late-exponential phase) grown in $300 \mathrm{~mL}$ or $100 \mathrm{~mL}$ of $\mathrm{M} 9$ mineral medium supplemented with PE or malate, respectively.

Each growth condition was established in triplicated and from each culture the total RNA was extracted according to the manufacturer's instructions of RNA Power Soil Total RNA Isolation Kit (Qiagen Italia, Italy) modifying the purification step that was performed by adding $3.5 \mathrm{~mL}$ of chloroform, mixing gently, centrifuging at $2500 \times \mathrm{g}$ for $10 \mathrm{~min}$, and transferring the upper phase to a clean tube. Then, the RNA was dissolved in $50 \mu \mathrm{L}$ of RNase-free water. RNA concentration was evaluated with 6000 Nano kit (Agilent, Italy). For each sample, RNA quality was measured using Bioanalyzer Agilent 2100 supported with RNA 6000 Pico Agilent chip (Agilent, Italy). RNA quality check showed an integrity number (RNI value) major of 7.5 for all the RNA samples.

RNA sequencing. The preparation of the sequencing library and the selective rRNA depletion to enrich the samples of mRNA transcripts were performed using Universal Prokaryotic RNA-Seq (Nugen, USA). The RNA sequencing of the samples was performed by Illumina HiSeq platform (Biodiversa, Italy) generating about 110 million and 113 million pairs of raw reads (forward and reverse strands) for the three malate samples and the three PE samples, respectively. The quality of raw sequencing data was checked with Trim Galore (version 0.4.4) (URL: http://www.bioinformatics.babraham.ac.uk/projects/trim_galore) to trim the sequencing reads ${ }^{26}$, while 
GPU-Dup Removal was applied to identify and remove duplicates ${ }^{27}$. The quality control resulted in about 92 million and 60 million reads for malate and PE samples, respectively. The sequences of RNA-seq are submitted to ENA with the following accession number PRJEB45685.

RNA-seq data analysis and differential gene expression. A reference-based strategy was used to assemble the transcriptome exploiting the genome sequences of $R$. opacus $\mathrm{R} 7$ previously obtained ${ }^{28}$. RNA-seq reads were mapped on R7 genome using TopHat aligner ${ }^{29}$ and they were separately processed and assembled by the Cufflinks default pipeline ${ }^{30}$ into a set of transcript fragments. The assembling process generated a total of 9679 transcripts with an overall read mapping rate of $54 \%$ and $85 \%$ for PE and malate samples, respectively. Then, the transcripts were merged in a format suitable for Cuffdiff software ${ }^{31}$ in order to obtain a set of loci with statistically different expression levels using default parameters. The final result was a table reporting the loci information (identifier, scaffold, start and stop location), the expression values (proportional to the number of reads mapping the locus), and the significance level (p-value) (Table S1). Lastly, the loci were ranked, filtered by $p$-value, and annotated by assigning the annotation of the corresponding Open Reading Frame (ORF). In addition, the expression values were adjusted by substituting the zero value of the expression with the minimum expression value of the dataset (excluding zero value).

Gene cluster annotation. A total of 9679 ORFs were predicted by Glimmer ${ }^{32}$ and annotated with Rapid Annotation using Subsystem Technology (RAST) ${ }^{33}$ resulting in function and subsystem classification. 9679 functionally annotated ORFs were grouped in subsystems that represent biological functional roles.

The differentially expressed genes (DEGs) were also manually analyzed using BLAST ${ }^{34}$ with e-value $<10$ and Clustal Omega ${ }^{35}$ against reference sequences from the UniProt database ${ }^{36}$ and literature searches.

Data resources used in gene cluster annotation include: $\mathrm{KEGG}^{37}$ and $\mathrm{GO}^{38}$. For each KEGG and GO term, the count of genes has been computed and compared between the two growth conditions.

Quantitative real-time PCR (RT-qPCR). The transcriptional induction of the R. opacus R7 genes potentially encoding enzymes involved in PE degradation was evaluated by quantitative reverse transcription (RT-) qPCR experiments. The amplified genes encode multicopper oxidases, LMCO1, 2, 3, and alkB gene and 16S rRNA used as a reference.

The reverse transcription of the total RNA was performed with iScriptcDNA Synthesis kit (BIO-RAD, Italy) to obtain $200 \mathrm{ng}$ cDNA with the following thermocycling conditions: $5 \mathrm{~min}$ at $25^{\circ} \mathrm{C}$ followed by $1 \mathrm{~h}$ at $42^{\circ} \mathrm{C}$ and then 5 min at $85^{\circ} \mathrm{C}$.

The amplification of the cDNA obtained was carried out using the StepOnePlus Real-Time PCR System (Applied Biosystem, Italy) in 10- $\mu \mathrm{L}$ qPCR volume containing $4.4 \mu \mathrm{L}$ of the reverse-transcribed RNA samples, $5 \mu \mathrm{L}$ of PowerUp SYBR Green Master Mix (Applied Biosystem, Thermo Scientific, Italy), and $300 \mathrm{nM}$ of each primer listed in Table S2. The temperature program was as follows: $30 \mathrm{~s}$ at $95^{\circ} \mathrm{C}$, followed by 40 cycles of $5 \mathrm{~s}$ at $95^{\circ} \mathrm{C}, 10 \mathrm{~s}$ at $60^{\circ} \mathrm{C}$ and $45 \mathrm{~s}$ at $72^{\circ} \mathrm{C}$ and one cycle of $15 \mathrm{~s}$ at $95^{\circ} \mathrm{C}, 1 \mathrm{~min}$ at $60^{\circ} \mathrm{C}$ and $15 \mathrm{~s}$ at $60^{\circ} \mathrm{C}$.

The expression of $16 \mathrm{~S}$ rRNA was used as a reference ${ }^{39}$ to normalize the tested gene expression according to the $\Delta \Delta \mathrm{Ct}$ method $^{40}$. The malate growth condition was used as a reference in order to determine the relative abundance of target transcripts. Data are expressed as mean \pm standard deviation derived from at least three independent experiments.

In order to exclude DNA contamination, negative controls were performed by omitting the reverse transcriptase in RT-PCR experiments, which were conducted with the same temperature program and the same primer sets for 35 cycles of amplification.

Bioinformatics analysis for the enzyme characterization. Based on RNA-seq data, we considered the aa sequences of multicopper oxidases induced after growth on PE and predicted with GO annotation and KEGG as putative laccase proteins (E.C. 1.10.3.2). Moreover, they were analyzed with EFICAz program ${ }^{41}$ to verify their enzymatic classification (EC number) and compared with PROSITE ${ }^{42}$ patterns to identify the conserved specific laccase domains (PDOC00076) corresponding to:

G-x-[FYW]-x-[LIVMFYW]-x-[CST]-x- $\{P R\}-\{K\}-x(2)-\{S\}-x-\{L F H\}-G-[L M]-x(3)-[L I V M F Y W]$ and

$\mathrm{H}-\mathrm{C}-\mathrm{H}-\mathrm{x}(3)-\mathrm{H}-\mathrm{x}(3)-[\mathrm{AG}]-[\mathrm{LM}]$.

Moreover, laccase aa sequences were retrieved from Protein Data Bank ${ }^{43}$ and aligned against $R$. opacus $\mathrm{R} 7$ putative laccases (UniProt ID: I6WZK7 and Q02219) to find the 3D structures with high similarity (more than $25 \%$ of percent identity).

In addition, the location of the correspondent protein was investigated predicting the presence of Twin-arginine signal peptide (Tat) by performing analyses through TATFIND v.1.4 ${ }^{44}$ (http://signalfind.org/tatfind.html), TatP v.1.0 ${ }^{45}$, SignalP-5.0 ${ }^{46}$ (http://www.cbs.dtu.dk/services/SignalP-5.0/index.php) and comparing their results.

Moreover, in order to evaluate the gene candidates retrieved from the RNA-seq analysis BLASTx tool of NCBI pipeline $^{34}$, Clustal Omega ${ }^{35}$, and $\mathrm{KEGG}^{37}$ were used to determine and compare amino acid sequence homology and to make manual curation.

Enzymatic assay. After R7 strain growth on PE or malate, a cell-free fraction (CFS) was obtained by centrifugation at $7000 \mathrm{rpm}$. Residual PE was removed by filtration with $0.45 \mu \mathrm{m}$ filters (Millipore). The supernatant was lyophilized and the product originating from $100 \mathrm{~mL}$ of $\mathrm{R} 7$ culture was redissolved in $2 \mathrm{~mL} 50 \mathrm{mM}$ potas- 
A

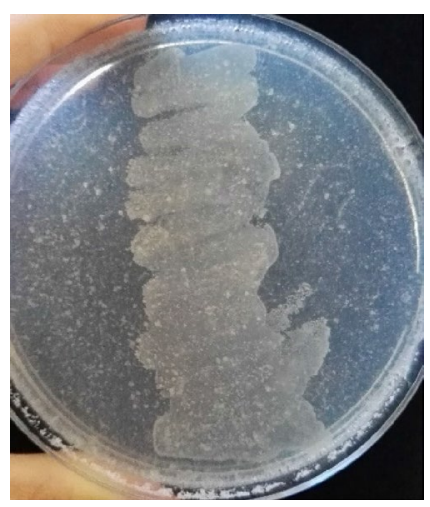

B

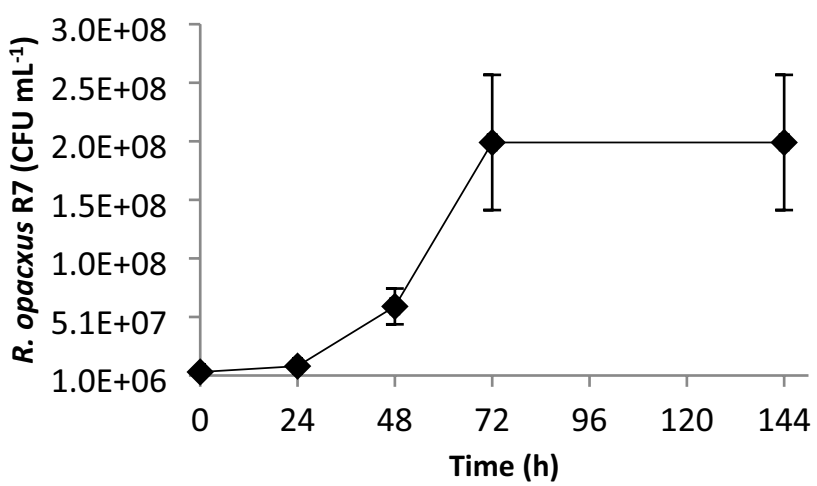

Figure 1. R. opacus R7 growth on polyethylene. (A) Shows preliminary growth assay performed inoculating R. opacus R7 on M9 mineral medium agar plates supplemented with 1.2\% PE and 1\% Tween80. (B) Shows R7 kinetic growth on M9 mineral medium supplemented with PE $1.2 \%$ as the only carbon and energy source.

sium phosphate buffer ( $\mathrm{pH}$ 7). This resuspension was dialyzed overnight against $400 \mathrm{~mL}$ of $0.2 \mathrm{mM}$ potassium phosphate buffer ( $\mathrm{pH} 7)$.

The specific activity of the lyophilized and dialyzed CFS fraction was determined in $50 \mathrm{mM}$ potassium phosphate buffer ( $\mathrm{pH} 7$ ). Laccase specific activity was defined as the amount of protein required to oxidize $30 \mathrm{mM}$ of 2,6-dimethoxyphenol (DMP) by increasing absorbance at $470 \mathrm{~nm}$ during 5-min intervals, for up to $60 \mathrm{~min}$. Laccase activity tests were carried out in a UV/visible spectrophotometer (Ultraspec 2100 pro, Amersham Biosciences). The specific activity was calculated as $\mathrm{U} \mathrm{mg}^{-1}$. The total protein concentration of the lyophilized and dialyzed CFS was assessed using the method of Bradford ${ }^{47}$ using Coomassie brilliant blue with bovine serum albumin as a standard. Protein concentrations were calculated from the standard curve by $20 \mu \mathrm{g} \mathrm{mL} \mathrm{m}^{-1}$ bovine albumin serum.

The activity and stability of R7 CFS were compared to the commercial fungal T. versicolor laccase $\left(\geq 0.5 \mathrm{U} \mathrm{mg}^{-1}\right.$ ) (Sigma-Aldrich, Italy).

Fourier transform infrared for the characterization of $R$. opacus R7 cells grown on polyethylene. R. opacus R7 cells grown on M9 mineral medium supplemented with $1.2 \%$ PE, 20 mM malate, or M9 mineral medium until the late-exponential phase were washed four times in physiological solution $(0.9 \% \mathrm{NaCl}$ $\mathrm{w} / \mathrm{v}$ in sterile Milli-Q water) at $8000 \mathrm{rpm}$ for $5 \mathrm{~min}$.

After resuspension of cell pellets in few microliters of $0.9 \% \mathrm{NaCl}, \sim 2 \mu \mathrm{L}$ of the resulting solutions were deposited onto a BaF2 window and dried at room temperature for about $30 \mathrm{~min}$ to eliminate the excess water.

FTIR absorption spectra were acquired in transmission mode, between 4000 and $700 \mathrm{~cm}^{-1}$, by the infrared microscope, Varian 610-IR coupled to the Varian 670-IR FTIR spectrometer (Varian Australia Pty Ltd., Mulgrave, VIC, Australia), equipped with a mercury cadmium telluride nitrogen-cooled detector. The variable microscope aperture was adjusted to $100 \mu \mathrm{m} \times 100 \mu \mathrm{m}$. Measurements were performed at $2.0 \mathrm{~cm}^{-1}$ spectral resolution, $25 \mathrm{kHz}$ scan speed, triangular apodization, and by the accumulation of 512 scan co-additions.

When necessary, the spectra were corrected for the residual water vapor and then normalized at the Amide I band area. The second derivative analysis was performed by the Savitzky-Golay method (3rd polynomial, 9 smoothing points), after a 13-point smoothing of the measured spectra, using the GRAMS/32 software (Galactic Industries Corporation, Salem, NH, USA).

For each sample, several measurements were performed by selecting different areas on the same sample (7-10 spectra for each condition in each experiment). Moreover, the reproducibility of the results was evaluated with three independent experiments.

\section{Results}

Growth of $R$. opacus R7 on polyethylene. $R$. opacus strain R7, which was selected for its ability to degrade numerous aliphatic, mono- and polycyclic aromatic hydrocarbons, and cyclo-carboxylic acids ${ }^{24}$, was preliminarily tested for the ability to grow on PE by a plate agar assay dissolving the PE powder in $1 \%$ Tween 80 . As shown in Fig. 1A, in the area of R7 cell deposition, the strain was able to grow in the presence of PE as the only carbon and energy source.

Consequently, a growth assay of R. opacus strain R7 was carried out in liquid M9 mineral medium culture supplemented with $1.2 \%$ PE up to $144 \mathrm{~h}$. R7 ability to degrade PE was evaluated by the colony-forming unit method over time (Fig. 1B). The result showed that the amount of R7 cells increased almost two orders of magnitude in $48 \mathrm{~h}$ and the maximum growth resulted in a total cell amount of around $2 \times 10^{8} \mathrm{CFU} \mathrm{mL}^{-1}$ at $72 \mathrm{~h}$.

Moreover, the modifications of content and structure of whole R7 cell were evaluated in the presence of polyethylene compared to malate condition by FTIR microspectroscopy (as reported in the paragraph FTIR characterization of $R$. opacus $\mathrm{R} 7$ intact cell grown on polyethylene). 
Overview of transcriptomic data from $R$. opacus $\mathrm{R} 7$ grown on polyethylene. The biodegradative mechanism of polyethylene by $R$. opacus R7 was studied through a transcriptomic approach performed after $72 \mathrm{~h}$ of R7 growth on PE by RNA-seq technology. R7 grown in the presence of malate was used as a reference for the transcriptomic analysis.

A reference-based strategy was used to assemble the transcriptomes and it produced a total of 9679 transcripts with an overall read mapping rate of 54\% and $85 \%$ for PE and malate samples, respectively. Quality control produced a total of 92 and 60 million reads for PE and malate conditions, respectively. The differential gene expression was obtained by calculating the $\log _{2}$ (fold_change) that from now is named LogFC, for the two different growth conditions.

Among the total number of R7 coding DNA sequences (CDSs), 97\% were not showing appreciable changes of gene expression under both conditions, while considering $p$-value $<0.05,130(1.3 \%)$ and $196(2 \%)$ genes were up- and down-regulated, respectively. The genes with a LogFC $>2$ (and p-value $<0.05$ ) were only $2.6 \%$ for both conditions. Overall, the up-regulated genes ( $\mathrm{p}$-value $<0.05$ ) showed a LogFC varying from 2 to 21.6 while the down-regulated genes from -2 to -20 (Table S1).

Abnormal LogFC values were shown by 19 differentially expressed genes (DEGs) due to the switch-off/-on effect $^{48}$, thus they were corrected with a min-value approximation resulting in an approximated LogFC varying from -20 to 21 . Among the 19 DEGs with corrected LogFC, 5 were up-regulated genes, 14 were down-regulated genes, and 11 genes were preliminary annotated as hypothetical proteins (Table S1).

The DEGs were variously distributed on R. opacus R7 genome that is constituted by one chromosome and five plasmids (pPDG1, pPDG2, pPDG3, pPDG4, and pPDG5): the highest number of up-regulated genes are located on the chromosome and pPDG1 and pPDG2 plasmids with $87 \%$ and $6 \%$ DEGs with respect to the total number of up-regulated genes, respectively. Instead, no up-regulated gene was reported on pPDG3, pPDG4, and pPDG5 plasmids. The down-regulated genes were predominantly located on R7 chromosome (83\%) and, $3 \%, 4 \%, 10 \%$, and $1 \%$ DEGs with respect to the total number of down-regulated genes were present on pPDG1, pPDG2, pPDG3, and pPDG4 plasmids, respectively. Notably, no down-regulated genes were recorded on the pPDG5 plasmid.

After Glimmer prediction, 9679 ORFs were functionally annotated with RAST. Afterward, the transcripts were further classified by GO and KEGG database using BLAST search against UniProt. In total, the annotation process allowed the classification of transcripts by RAST (9664 total number), GO (8991 total number), and KEGG (3416 total number) databases.

Figure 2 represents the number of R7 DEGs associated with the main GO categories, such as organic substance catabolic process, dioxygenase, monooxygenase, hydrolase activity, and transport. Out of 301 assigned DEGs to GO categories 271 were assigned to "molecular functions", 266 to "biological processes", and 178 to "cellular components". The highest percentage of transcripts were classified under the following GO categories: $74 \%$ of DEGs under GO annotation as "metabolic processes", $68 \%$ as "catalytic activity", and $66 \%$ as "cellular processes".

The transcripts were also classified by KEGG identifiers exhibiting 384 DEGs associated with 343 pathways. Among the KEGG-annotated sequences, 70 were grouped under the "metabolism" category ( $63 \%$ of total DEGs), 23 under "environmental information processing" (21\%), 22 under "genetic information processing" (20\%), 6 under "cellular processes" identifier (5\%), and 2 under "organismal systems" (3\%) (Fig. 3). More in detail, 13 into "biosynthesis of various secondary metabolites", 13 into "microbial metabolism in diverse environments", 6 into "carbon metabolism", and 1 into "ABC transporters".

Overall, the transcriptome showed DEGs associated with oxidoreductase activity, hydrocarbon catabolic processes, central metabolism, transporters, and membrane activity. On the other hand, the analyses also showed that $15 \%$ of the positively regulated genes and $18 \%$ of the negatively regulated genes were preliminary annotated as hypothetical proteins (HP) with unknown functions with respect to total DEGs (Table S1).

Based on the classification of GO and KEGG databases, differentially regulated genes connected to stress and translation were also identified. Specifically, eleven differentially regulated genes related to stress including among the other genes putatively encoding a xylose repressor XylR (ROK family), a flavohemoprotein (hemoglobin-like protein) (flavohemoglobin) (nitric oxide dioxygenase) (EC 1.14.12.17), a thiol peroxidase, Bcp-type (EC 1.11.1.15). a L-ectoine synthase (EC 4.2.1.-), a possible universal stress protein, a stress responsive A/B Barrel Domain superfamily protein, and a heat shock protein 60 family chaperone GroEL were detected in the RNA-seq. On the other hand, few genes related to stress response were down-regulated including genes encoding proteins for DNA recombination and repair, L-proline biosynthetic process and response to copper ion. Moreover, nine up-regulated genes showed a GO annotation related to translation including six annotated as hypothetical proteins, and a collagen triple helix repeat, a TsaC protein (YrdC domain) required for threonylcarbamoyladenosine t(6)A37 modification in tRNA, and a LSU ribosomal protein L11p (L12e). On the other hand, two down-regulated genes annotated as hypothetical proteins showed a GO annotation related to translation.

Identification of specific pathways induced in R. opacus R7 grown on polyethylene. In order to identify the genetic determinants involved in polyethylene degradation by $R$. opacus strain R7, the transcriptomic data were explored searching for genes potentially involved in the biodegradative pathway, including genes encoding oxygenases and oxidases.

Accordingly, RNA-seq data highlighted a R. opacus R7 gene, LMCO1 (AII08809) that was described as a multicopper oxidase by the preliminary annotation and was up-regulated 19.5-fold. LMCO1 gene is located on the chromosome downstream a genome region comprising genes encoding a benzoate dioxygenase system and two-component monooxygenase [(4-hydroxyphenylacetate 3-monooxygenase (EC 1.14.13.3) and nitrilotriacetate monooxygenase component B (EC 1.14.13.-)] (PheA1). 


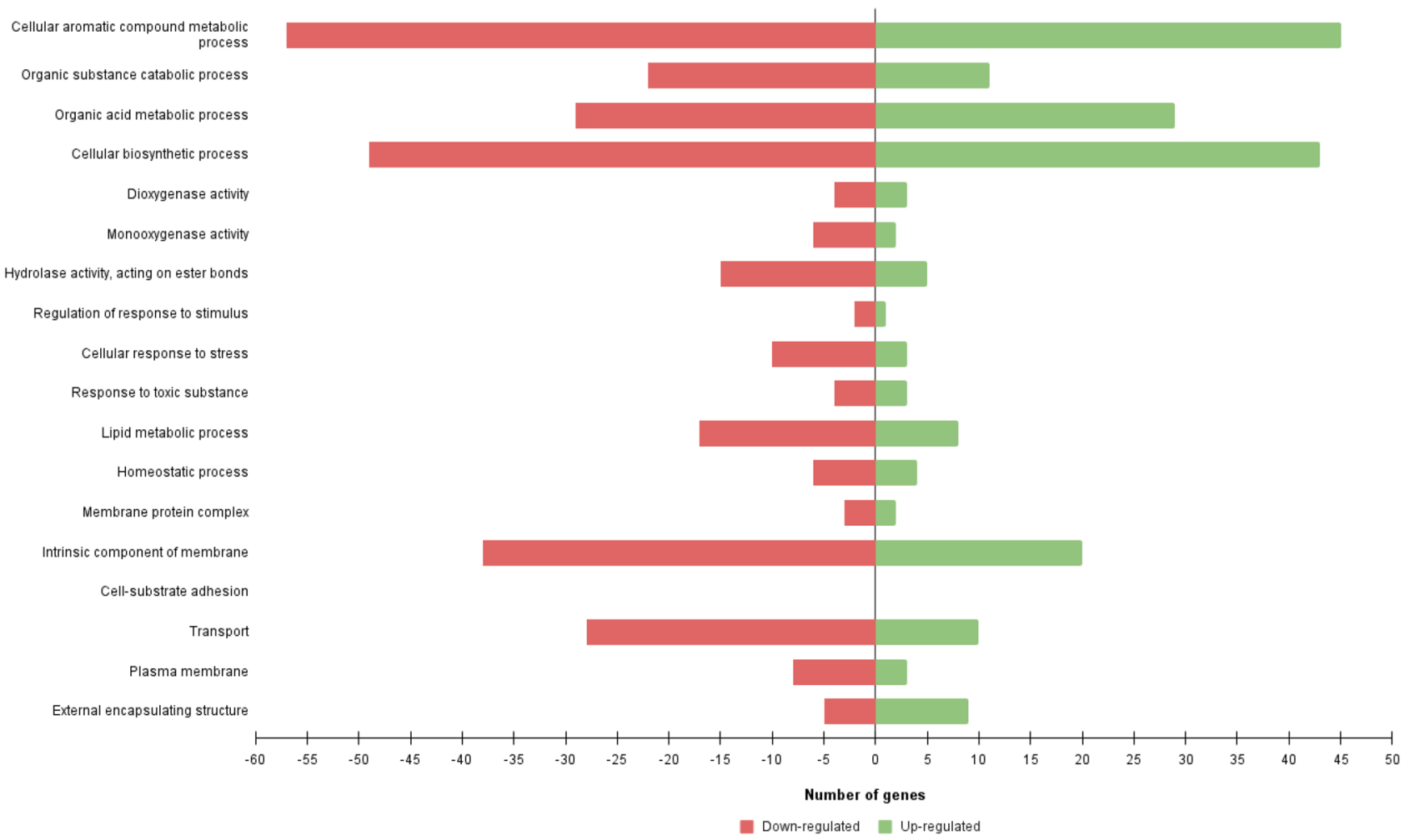

Figure 2. Distribution of R. opacus R7 DEGs respect with polyethylene and malate growth conditions according to physiological categories. Barplot shows the number of up- and down-regulated genes associated with GO categories such as cellular aromatic compound metabolic processes, organic substance catabolic processes, organic acid metabolic processes, cellular biosynthetic processes, dioxygenase activity, monooxygenase activity, hydrolase activity, acting on ester bonds, regulation of response to stimulus, cellular response to stress, response to toxic substance, lipid metabolic processes, homeostatic processes, membrane protein complex, intrinsic component of membrane, cell-substrate adhesion, transport, plasma membrane, external encapsulating structure.

Genetic Information Processing

$$
\text { Cellular Processes }
$$

Organismal Systems

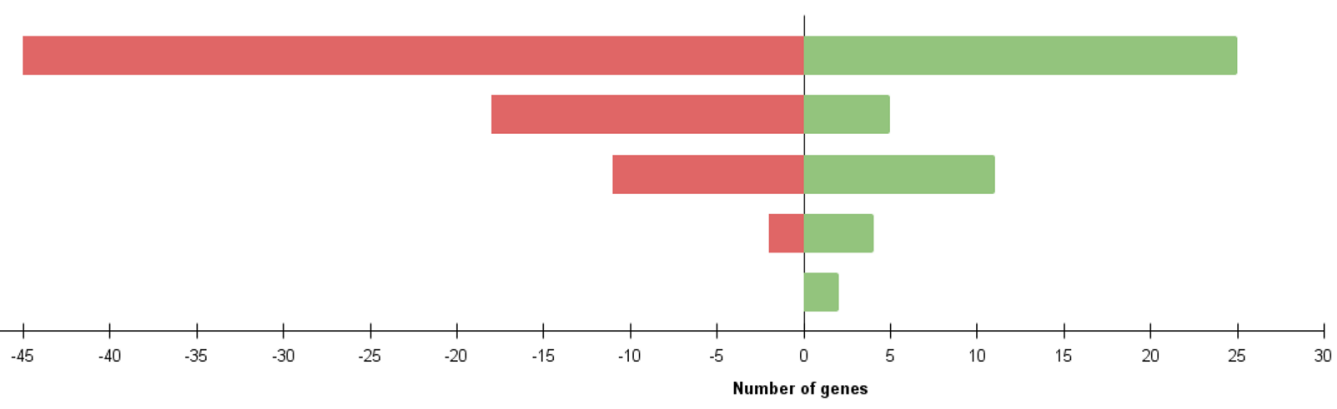

Down-regulated Up-regulated

Figure 3. Number of up- and down-regulated genes of R. opacus R7 in response to polyethylene and malate according to KEGG identifiers. Distribution of the main KEGG classes including metabolism, environmental information processing, genetic information processing, cellular processes, and organismal systems.

Among R7 oxidases, the other two multicopper oxidases were considered although the RNA-seq analysis did not show evidence of their induction. They are located on pPDG3 plasmid: LMCO2 gene (AII11185) is located in a genome region including genes encoding (copper-) membrane transporters; LMCO3 gene (AII11221) is located just down-stream genes encoding copper resistance genes and up-stream carbonic anhydrase, and zinctransporter genes.

In order to bioinformatically analyze the three R7 multicopper oxidases, LMCO aa sequences were compared to the laccase-like enzyme sequence identified in $R$. ruber C208 genome for its role in PE degradation. The alignments (performed on the NCBI pipeline) showed that the amino acidic identity of C208 laccase was $48 \%$ with respect to LMCO1, 53\% with respect to LMCO2, and 23\% with respect to LMCO3. Moreover, R7 LMCO aa sequences were aligned vs NCBI pipeline identifying all the copper-binding sites characteristic of laccase 
enzymes $^{49,50}$ in two of the three R7 oxidases (LMCO1 and LMCO2), while only two copper-binding sites were identified in LMCO3 (Fig. 4A). R7 LMCO aa sequences were further characterized with EFICAz software and PROSITE pattern search that confirmed that two aa sequences were predicted as putative multicopper oxidases (LMCO1 and LMCO2) which also showed more than $25 \%$ of sequence identity with respect to a "Crystal structure of Lac15 from a marine microbial metagenome" (PDB structure 4F7K) as shown in Fig. 4B,C. The other aa sequence (LMCO3) does not have a specific known laccase domain but shows $57 \%$ of sequence identity with respect to the crystal structure of a reference multicopper oxidase (PDB structure: 3GDC).

In addition, R7 aa LMCO sequences were screened for signal amino acids (aas) for the translocation of folded proteins across lipid bilayers ${ }^{51}$. All three R7 sequences evidenced conserved twin-arginine (TAT) signal peptide motif consisting of (S/T)RRxFL (with $x$ referring to a polar amino acid) and a hydrophobic region of an average length among 18 and 22 amino acids, located downstream TAT signal aas and up-stream the predicted cleavage peptides.

After the initial oxidative step of polyethylene through the secretion of extracellular oxidases, efficient mineralization of polyethylene would require additional steps, including (i) specific transport systems that can be hypothesized for short length-oxidized-PE fragments, (ii) additional cytoplasmic oxidations to finalize small $\mathrm{PE}$ fragments oxidation, and (iv) the subsequent entrance of these fragments into the beta-oxidation pathway.

Therefore, based on this hypothesis, 5 different transcripts (AII08802, AII09363, AII08803, AII03285, and AII08421) encoding oxygenases/hydroxylases were considered for their induction during growth on PE, showing a LogFC ranging from 4 to 19.4 (Table S1, Table S3). Among these up-regulated genes, three genes encode respectively a sarcosine oxidase delta subunit, a 2-polyprenylphenol hydroxylase, and related flavodoxin oxidoreductases, and a conserved hypothetical hydroxylase, putatively involved in R7 stress response; one benzoate dioxygenase gene probably activated for its role in the central metabolism, and only one gene coding for a putative cytochrome P450 hydroxylase (AII08421) (Table S3). AII08421 was similar to cytochrome P450 hydroxylase CYP124 of Mycobacterium spp. with an aa identity of 32\% (PDB: 2WM4, 6T0F, and 6CVC). Moreover, amino acid sequence comparison between R7 P450 hydroxylase and CYP125 of Rhodococcus erythropolis PR4 (RER_33720) or Rhodococcus jostii RHA1 (WP_009477746) showed 31\% and 29\% identity, respectively ${ }^{52}$. Therefore, the preliminary bioinformatics analyses of AII08421 amino acid sequence revealed that it could be included in CYP124 or CYP125 families. Indeed, the analysis of CYP124A1 substrate specificity for Mycobacterium strains indicated that it catalyzes the terminal hydroxylation of methyl-branched hydrocarbon chains ${ }^{53}$; cyp125 genes of R. erythropolis PR4 are activated in the presence of diesel oil, while CYP125 of $R$. jostii RHA1 is fundamental to initiate the catalysis of 3-hydroxysterols (cholesterol) via sterol side-chain degradation ${ }^{54}$. Overall, these preliminary suggestions indicated the possible involvement of R7 cytochrome P450 gene in the oxidation of internalized PE short chains.

Likewise, there is a redundancy of genes in R7 genome encoding other monooxygenases/hydroxylases. For example, we considered the alkane-1-monooxygenase encoding gene since its involvement in the degradation of medium-chain $n$-alkanes $\left(\mathrm{C}_{12}\right)$ has been verified in the previous work ${ }^{55}$. $\mathrm{R} 7$ alkB (AII08632) is a unique gene in the genome and it is expressed during R7 growth on PE. AlkB is also reported to be involved in PE degradation since it is induced by medium-chain alkanes ${ }^{15,20,21}$.

Consistent with this hypothesis, diverse R7 genes putatively encoding membrane protein (AII10792), integral membrane protein (AII10609), and putative ATP/GTP-binding integral membrane protein (AII04246) appeared up-regulated during the R7 PE degradative process with LogFC 5.5, 3, and 2, respectively. Amino acid sequence alignment of these three membrane proteins against NCBI pipeline showed $67 \%, 84 \%$, and $88 \%$ identity with DUF3556 domain-containing protein, DUF475 domain-containing protein, and ATPase. In addition, other gene candidates were retrieved from genome analysis such as genes encoding an ABC transporter ATP-binding protein (AII09203), a protein-export membrane protein SecD (TC 3.A.5.1.1) (AII04221), and other membrane proteins (AII06740 and AII07038) which could have a role in PE metabolism.

Finally, the PE mineralization can be achieved through the $\beta$-oxidation pathway. Indeed, R7 genes putatively involved in this pathway were up-regulated with LogFC varying between 18 and 4 under PE growth as observed by $^{15}$. Indeed, the metabolism of short-medium chain alkanes usually proceeds through a (NAD(P)-dependent) alcohol dehydrogenase (AII08419), aldehyde dehydrogenase (EC 1.2.1.3) (AII08418), acetaldehyde dehydrogenase (EC 1.2.1.10) (AII03621), long-chain-fatty-acid-CoA ligase (EC 6.2.1.3) (similar to WP_128639699), 3-hydroxybutyryl-CoA dehydrogenase (EC 1.1.1.157)/3-hydroxyacyl-CoA dehydrogenase (EC 1.1.1.35) (AII05967).

RT-qPCR analysis of selected $R$. opacus R7 genes from RNA-seq. RT-qPCR experiments were performed on cDNA deriving from R7 cell grown in the presence of PE or malate in order to validate the transcription levels of R7 candidate genes putatively involved in PE degradation. We selected genes encoding oxidases and oxygenases that could be good candidates for the two main oxidative steps of PE degradation: the first oxidation on the cellular membrane or in the extracellular environment and the cytoplasmic oxidation of PE when shorter fragments of polymer are internalized. Therefore, we selected LMCO1 (AII08809), LMCO2 (AII11185), LMCO3 (AII11221) genes encoding multicopper oxidases, and alkB (AII08632) gene encoding an alkane-1-monooxygenase. Figure 5 shows the values of RT-qPCR activation obtained with the $\Delta \Delta \mathrm{Ct}$ method for each selected candidate gene.

The gene expression evaluated by RT-qPCR showed identical trends in terms of the direction of differential expression (up-regulation) for all the tested genes with respect to RNA-seq.

Enzyme assay on $R$. opacus R7 supernatant. Based on RNA-seq data and preliminary bioinformatic analyses, we hypothesized that $R$. opacus R7 grown on PE produces an extracellular degradative enzyme system 


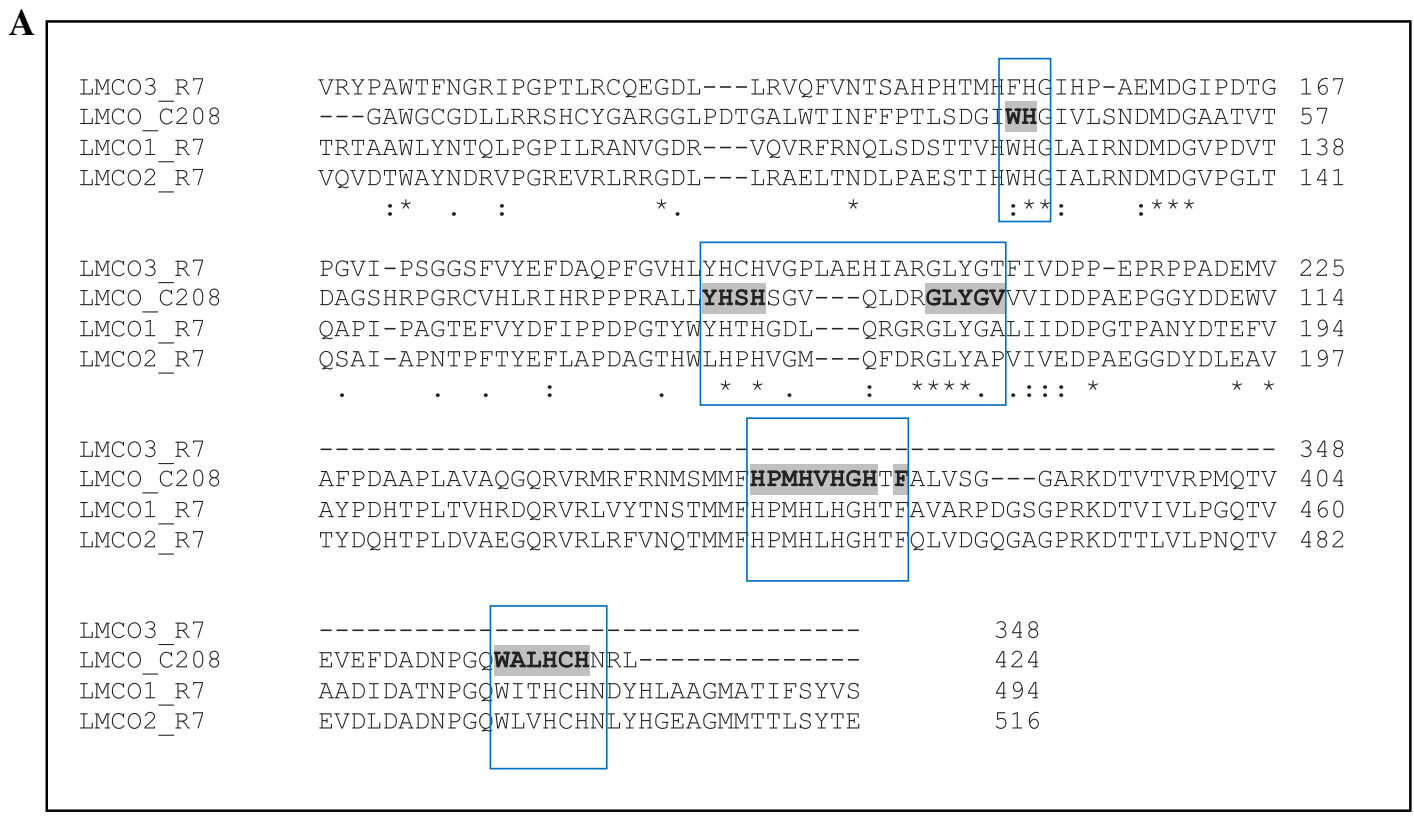

B

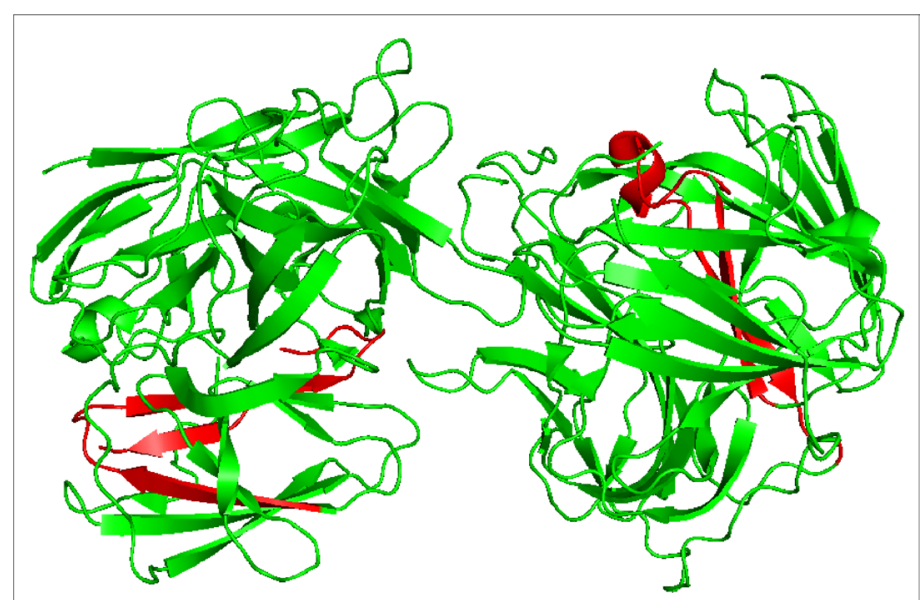

C

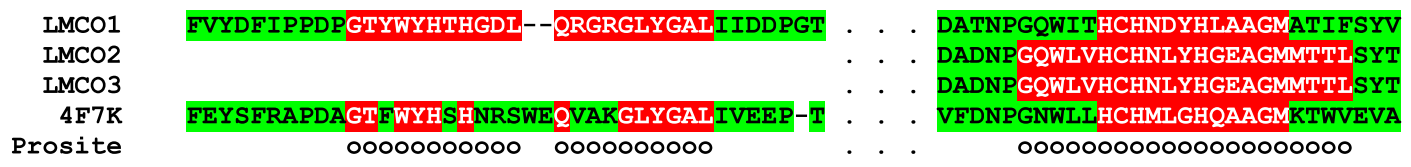

Figure 4. Bioinformatics analyses of amino acid sequences of R. opacus R7 LMCO gene products encoded by LMCO1, 2, and 3 DEGs. Multiple alignment of LMCO1, 2, and 3 amino acid sequences with respect to $R$. ruber C208 LMCO sequence evidencing putative copper-binding motifs generally associate to the class of laccases (rectangular area); bold grey amino acids correspond to the copper-binding motifs reported for C208 sequence ${ }^{22}$ (A). Protein structure of Lac15 from a marine microbial metagenome (PDB 4F7K) showing in red color the laccase domain regions (B). Multiple alignment of the conserved domain region of 4F7K sequence respect with LMCO1, 2, and 3 amino acid sequences according to EFICAz software and PROSITE (C).

similar to laccase-like. Therefore, the laccase-like activity was monitored collecting the CFS at maximum growth level of $R$. opacus $\mathrm{R} 7$ on PE after $72 \mathrm{~h}$. The enzymatic activity of R7 CFS reached a maximum of $10^{-6} \mathrm{U} \mathrm{mg}^{-1}$. Additionally, the corresponding CFS was concentrated 50 and 500 times without significant loss of activity.

Moreover, the laccase activity was assessed on CFS isolated from R7 culture on malate and it was equal to $6 \times 10^{-8} \mathrm{U} \mathrm{mg}^{-1}$. Notably, the laccase-like activity evidenced in the supernatant without cells (CFS) of R7 grown on PE was higher with respect to the CFS obtained from R7 grown on malate. We can speculate that this laccase-like activity could correspond to the activity exerted by LMCO genes that were up-regulated in the transcriptomic analysis. 


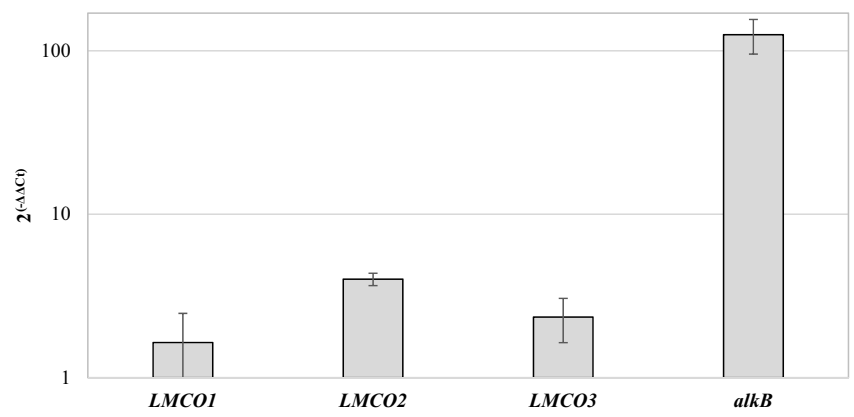

Figure 5. Expression levels of R7 selected genes for RT-qPCR analysis. The selected genes are LMCO1, $L M C O 2, L M C O 3$, and alkB encoding laccase-like multicopper oxidase 1, 2 and 3 and alkane monooxygenase, respectively.
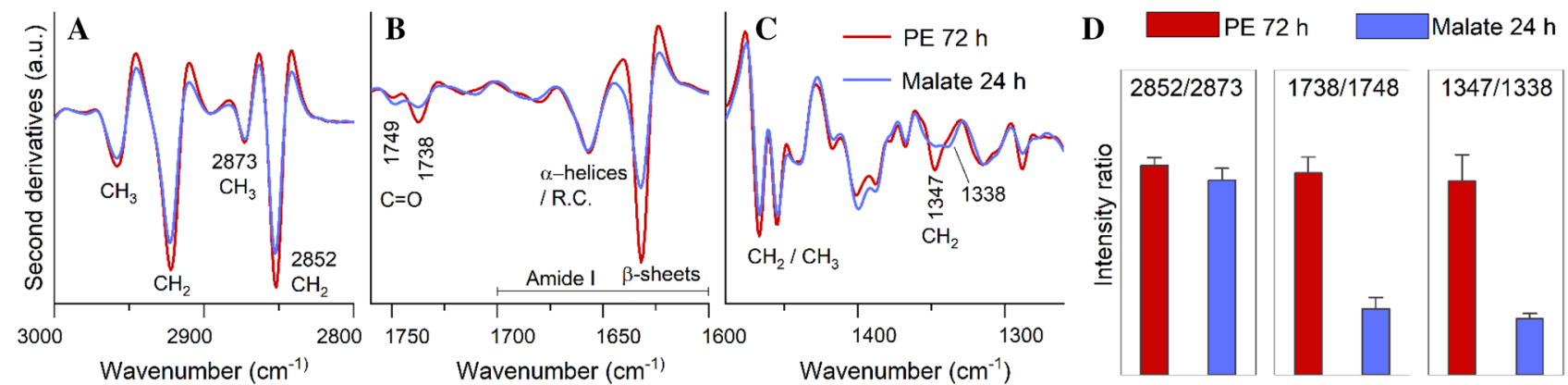

Figure 6. FTIR analysis of R. opacus R7 intact cells. The second derivatives of the mean FTIR absorption spectra of $R$. opacus $\mathrm{R} 7$ cells grown on PE for $72 \mathrm{~h}$ and malate for $24 \mathrm{~h}$ are reported in the spectral ranges: (A) $3000-2800 \mathrm{~cm}^{-1}$, (B) $1760-1600 \mathrm{~cm}^{-1}$, (C) $1500-1250 \mathrm{~cm}^{-1}$. The peak position and the assignment at selected vibrational modes are indicated. Panel (D) shows the intensity ratios between the reported peaks $\left(2852 \mathrm{~cm}^{-1} / 2873 \mathrm{~cm}^{-1}, 1738 \mathrm{~cm}^{-1} / 1748 \mathrm{~cm}^{-1}, 1347 \mathrm{~cm}^{-1} / 1338 \mathrm{~cm}^{-1}\right)$, taken in the second derivative spectra. The error bars represent the standard deviation from three independent experiments.

FTIR characterization of $\boldsymbol{R}$. opacus R7 intact cells grown on polyethylene. To disclose possible modifications in the content and the structure of the whole R. opacus R7 cell biomolecules induced by PE as the only carbon and energy source, compared to malate condition, FTIR microspectroscopy was employed. This vibrational approach enables to characterize not only isolated biomolecules but also complex biological systems, such as intact cells, providing a snapshot of the structure and content of the main molecules ${ }^{56}$. For sake of clarity, only the spectral bands displaying the most important spectral differences in the two growth conditions will be described. In particular, we analyzed the second derivative spectra in the spectral ranges where the functional groups of the main biomolecules absorb (Fig. 6A-C).

Between $3000-2800 \mathrm{~cm}^{-1}$ (Fig. 6A), the second derivative spectra are characterized by four well resolved bands assigned mainly to lipid hydrocarbon chain vibrational modes: $\sim 2958 \mathrm{~cm}^{-1}\left(\mathrm{CH}_{3}\right.$ asymmetric stretch), $\sim 2922 \mathrm{~cm}^{-1}\left(\mathrm{CH}_{2}\right.$ antisymmetric stretch), $\sim 2873 \mathrm{~cm}^{-1}\left(\mathrm{CH}_{3}\right.$ symmetric stretch), and $\sim 2852 \mathrm{~cm}^{-1}\left(\mathrm{CH}_{2} \mathrm{sym}-\right.$ metric stretch $)^{57}$. In particular, the $\mathrm{CH}_{2}$ bands display a higher intensity in the PE cell spectrum compared to control cells (Fig. 6A,D). In the spectral range 1760-1600 $\mathrm{cm}^{-1}$, the second derivative spectra show two peaks mainly ascribable to carbonyl groups involved in ester bonds ${ }^{56}$, at $\sim 1749 \mathrm{~cm}^{-1}$ and $\sim 1738 \mathrm{~cm}^{-1}$, with a different intensity in the two conditions (Fig. 6B,D). In addition, also the Amide I band components, due to the $\mathrm{C}=\mathrm{O}$ stretch of the peptide bond and giving information on protein secondary structures, are observed at $\sim 1657 \mathrm{~cm}^{-1}$ (assigned to protein alpha helix/random coil structures), and at $\sim 1632 \mathrm{~cm}^{-1}$ with higher intensity in PE cells (due to protein beta-sheets) (Fig. 6B) ${ }^{56}$.

Finally, in the spectral range $1500-1250 \mathrm{~cm}^{-1}$, absorption bands mainly assigned to hydrocarbon chain vibrational modes occur: the $\sim 1467 \mathrm{~cm}^{-1}$ (deformation modes of $\left.\mathrm{CH}_{2} / \mathrm{CH}_{3}\right)^{57}$ that, in agreement with what found between $3000-2800 \mathrm{~cm}^{-1}$, is of higher intensity in PE cells compared to control; a complex absorption between $\sim 1361$ and $1331 \mathrm{~cm}^{-1}$, which is assigned to hydrocarbon chain $\mathrm{CH}_{2}$ wagging vibrations $\mathrm{s}^{57}$. Here, in $\mathrm{PE}$ cells, the component at $\sim 1347 \mathrm{~cm}^{-1}$ is at higher intensity compared to control where, instead, also a component at $\sim 1338 \mathrm{~cm}^{-1}$, negligible in PE cells, is found (Fig. 6C,D).

Overall, the FTIR results indicate that $R$. opacus $\mathrm{R} 7$ growth on PE leads to spectral differences, compared to the growth on malate, involving mainly the response of lipids and proteins. These differences could reflect a different metabolism in the two cases. 


\section{Discussion}

The importance of addressing the issue of plastic disposal is nowadays of great relevance. Among the different kinds of plastic and plastic wastes, polyethylene has gained the center of attention due to its properties of non-degradability and durability ${ }^{3}$. Certainly, these features have ensured its large utilization and duration but on the other hand have posed the urgent problem of plastic waste disposal. In general, biodegradation is an ecofriendly approach to apply to different fractions of plastic wastes, thus more versatile with respect to chemical or mechanical processes.

Up to now, few bacterial genera have been characterized for the ability to degrade diverse types of $\mathrm{PE}^{20,21}$, at least partially, and only Rhodococcus ruber C208 has been investigated for the genetic determinants involved in this metabolism ${ }^{15}$.

Interestingly, R. opacus R7 is capable to grow in the presence of mineral medium supplemented with PE as the only carbon and energy source. R7 growth culture reached a high level of cell amount after $72 \mathrm{~h}$ with a growth profile similar to the one observed for R. ruber $\mathrm{C} 208^{15}$. Although the induction of the pathways related to alkane degradation and $\beta$-oxidation of fatty acids have been identified in C208 grown on PE, the complex gene framework involved in PE biodegradation is still unclear and not completely explored. Therefore, the present study aims at in-depth reconstruction of $R$. opacus R7 gene framework for PE degradation through the RNAseq approach evaluating the possible involvement of specific enzymatic systems. This work shows R. opacus R7 transcriptome analysis performed after three days of growth on PE to identify genes encoding candidate enzymes involved in PE degradation.

The RNA-seq approach allowed uncovering a total of 130 up-regulated genes (p-value $<0.05$ ) of $R$. opacus R7. Newsworthy, these up-regulated genes were mostly located on the chromosome (87\%). Although several transcripts were associated with hypothetical proteins (HP), the GO and KEGG analysis showed that several up-regulated transcripts were associated with oxidoreductase activity, hydrocarbon catabolic processes, central metabolism, transporters, and membrane activity; in addition, few DEGs related to stress or translation were also identified.

Considering oxidoreductase activity, specifically oxidative activity, R7 RNA-seq data together with RT-qPCR experiments highlighted three up-regulated genes annotated as multicopper oxidases (LMCO1, 2, 3). The predictive bioinformatics analyses indicated that these genes belong to "laccase-like multicopper oxidase" (LMCO) enzymatic class ${ }^{49}$. Moreover, the results of $R$. opacus R7 transcriptome are in line with the report of a laccase-like enzyme (EC 1.10.3.2) able to catalyze externally the biotic oxidation of PE and the suggestion that the same class of enzyme could be responsible for the first PE oxidation step before of smaller PE fragments are internalized in Rhodococcus cells ${ }^{15,22}$. The predictive bioinformatic analyses on R7 LMCO1, 2, and 3 gene sequences supported the idea of their involvement in PE metabolism since the correspondent amino acid sequences possess the main conserved pattern domains and the copper-binding motifs typical of enzymes possibly involved in PE degradation ${ }^{1}$.

In addition, enzymatic assays for laccase activity performed on R7 CFS collected after growth on PE added the credence that the detected laccase-like activity could correspond to the activity exerted by LMCO genes that were up-regulated in transcriptomic analysis. Moreover, the laccase-like activity detected in R7 CFS is comparable to the laccase activity of C208 strain without copper addition ${ }^{22}$.

The overall analysis of RNA-seq data showed also that other transcripts encoding oxygenases/oxidases were up-regulated and they could participate in further steps of intracellular PE-smaller fragment oxidation as reported by Yoon et al., Jeon and Kim, and Gravouil et al. ${ }^{15,20,21}$. Specifically, some authors have reported the involvement of an alk degradative system in the oxidation of internalized short-chain fragments of $\mathrm{PE}^{20,21}$. In R7 genome an alkB gene is present in a unique copy and it was previously studied for its involvement in mediumchain $n$-alkanes ${ }^{55}$. RT-qPCR expression experiments showed that R7 alkB gene is around 100-fold up-regulated under PE condition with respect to the reference condition which is in line with what was observed by Gravouil and coworkers ${ }^{15}$ for $\mathrm{C} 208$ strain.

Moreover, R7 transcriptome showed also an up-regulated gene encoding a cytochrome P450 (AII08421) similar to CYP124 of Mycobacterium spp., CYP125 of Rhodococcus erythropolis PR4 (RER_33720), and Rhodococcus jostii RHA1 (WP_009477746) with around 30\% amino acid identity, indicating that R7 P450 belongs to CYP124 or CYP125 families. These enzymatic classes catalyze the side-chain cholesterol degradation or are activated in the presence of diesel oil, thus, involved in the biodegradation of aliphatic carbon chains $\mathrm{s}^{52-54}$.

Overall, these preliminary suggestions indicated the possible involvement of R7 different oxidative systems in the degradation of internalized short-chain PE.

Finally, R7 PE mineralization can be achieved through the induction of genes for the $\beta$-oxidation pathway. Indeed, five up-regulated genes of R7 were identified as good candidates to accomplish the complete degradation of saturated fatty acids as observed by Gravouil and coworkers ${ }^{15}$.

Consistent with this hypothesis, among the overexpressed genes we looked for genes encoding transport systems. Diverse R7 genes encoding different membrane proteins (3 different genes), an integral membrane protein, a putative ATP/GTP-binding integral membrane protein, an ABC transporter ATP-binding protein, and a protein-export membrane protein $\mathrm{SecD}$ (TC 3.A.5.1.1) were activated during PE degradative process.

In order to confirm the ability of R7 to degrade PE and the consequent expression of specific genes, FTIR analysis was performed, and the results strongly suggested that PE growth condition compared to malate condition induces in R7 strain metabolic changes that involve mainly lipids and then proteins. Indeed, the most significant spectral differences between the two cases affect several absorption bands that are ascribable to $\mathrm{CH}_{2}$ groups of lipid hydrocarbon chains, which display a higher intensity in PE cell spectra. This result could indicate the presence of longer lipid hydrocarbon chains in the membranes compared to cells grown on malate, which affects the physical-chemical properties of cell lipids (for R7 cells grown on PE), as also supported by the spectral 

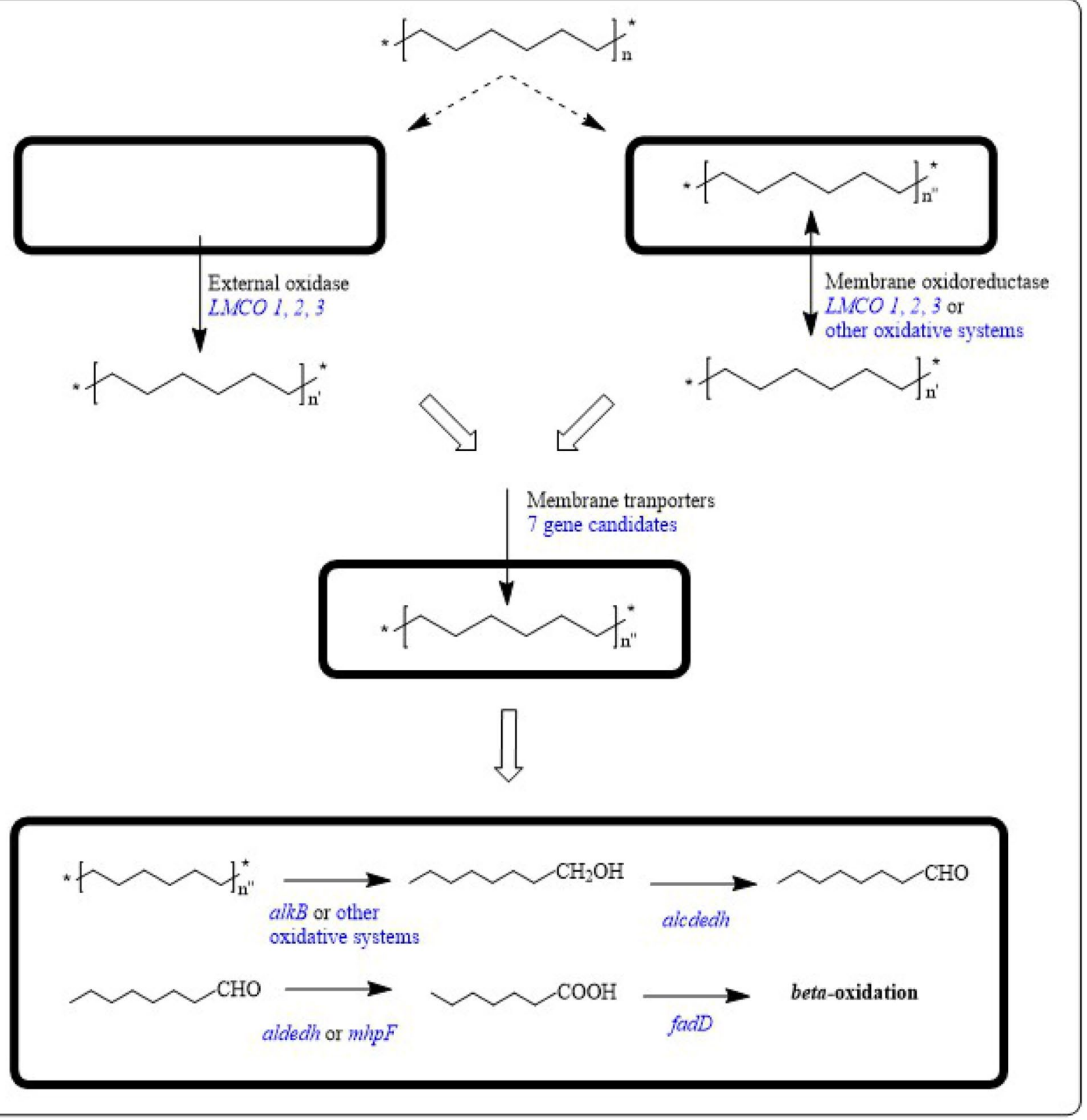

Figure 7. Proposed metabolic pathways of R. opacus R7 for polyethylene degradation. Blu genes represent candidate genes involved in PE metabolism: LMCO 1, LMCO 2, and LMCO 3, laccase-like multicopper oxidase; membrane oxidoreductase or other oxidative systems include alkB system; alkB, alkane monooxygenase; alkB or other oxidative systems include cyp450, cytochrome P450 hydroxylase. Membrane transporters include integral membrane proteins. alcdedh, alcohol dehydrogenase; aldedh, aldehyde dehydrogenase; $m h p F$, acetaldehyde dehydrogenase; $f a d D$, long-chain-fatty-acid-CoA ligase. Names of enzyme categories reported in black color represent candidates for each metabolic step. Dashed arrows indicate hypothetical metabolic steps. Full white arrows indicate required metabolic steps.

features of the $\mathrm{C}=\mathrm{O}$ band between $\sim 1760-1730$. This absorption is responsive to changes in the carbonyl group environment, including hydrogen bonding and polarity ${ }^{57}$. Interestingly, R7 cells grown on the two different carbon sources displayed significant spectral differences also in this range. Furthermore, the FTIR result highlighted differences in the overall cell protein secondary structures in the two conditions. Particularly, R7 cells grown in the presence of PE showed a more intense beta-sheet band compared to R7cells grown on malate.

In conclusion, this work provides evidence that Rhodococcus opacus R7 activates diverse oxidases and oxygenases to cope with PE as the only carbon and energy source. For the first time, the complete polyethylene degradative pathway is proposed. LMCO genes were up-regulated as the first step of oxidation upon PE outside exposure, indicating that this process is presumably performed on the cell membrane or on its interface with the extracellular environment. Consequently, more than one oxidoreductase system takes action on PE favoring 
the entrance of the smaller aliphatic fragments. Thus, R7 internally uses monooxygenases and hydroxylases to reduce alkane fragment length leading to the $\beta$-oxidation pathway (Fig. 7).

These findings are noteworthy, and they can be considered valuable to address the current plastic waste emergency in terms of a novel microorganism able to biodegrade polyethylene and above all new data on the genes involved in this degradation; as a matter of fact, the identification of these gene products poses the basis for diverse environmental and biotechnological applications contributing to plastic elimination. For example, they could be used as marker sequences for environmental biomonitoring as well as they can be useful as biotechnological catalysts for the biodegradation of polyethylene-based plastics.

Received: 6 July 2021; Accepted: 12 October 2021

Published online: 29 October 2021

\section{References}

1. Ghatge, S., Yang, Y., Ahn, J. H. \& Hur, H. G. Biodegradation of polyethylene: A brief review. Appl. Biol. Chem. 63, 27. https://doi. org/10.1186/s13765-020-00511-3 (2020).

2. PlasticsEurope (Ed.) Plastics-The Facts, 2019 ed. PlasticsEurope. https://www.plasticseurope.org/application/files/9715/7129/ 9584/FINAL_web_version_Plastics_the_facts2019_14102019.pdf (2018).

3. Scarascia-Mugnozza, G., Sica, C. \& Russo, G. Plastic materials in European agriculture: Actual use and perspectives. J. Agric. Eng. 3, 15-28 (2011).

4. Goldstein Market Intelligence. https://www.goldsteinresearch.com/report/global-polyethylene-market. Accessed 14 June 2021.

5. Restrepo-Flórez, J.-M., Bassi, A. \& Thompson, M. R. Microbial degradation and deterioration of polyethylene-A review. Int. Biodeterior. Biodegrad. 88, 83-90 (2014).

6. Song, J. H., Murphy, R. J., Narayan, R. \& Davies, G. B. H. Biodegradable and compostable alternatives to conventional plastics. Philos. Trans. R. Soc. B. 364, 2127-2139 (2009).

7. Dey, U., Mondal, N. K., Das, K. \& Dutta, S. An approach to polymer degradation through microbes. IOSR J. Pharm. 2, 385-388 (2012).

8. Krueger, M. C., Harms, H. \& Schlosser, D. Prospects for microbiological solutions to environmental pollution with plastics. Appl. Microbiol. Biotechnol. 99, 8857-8874 (2015).

9. Hou, L. et al. Biodegradability and ecological impacts of polyethylene-based mulching film at agricultural environment. J. Hazard. Mater. 378, 120774 (2019).

10. Albertsson, A.-C., Andersson, S. O. \& Karlsson, S. The mechanism of biodegradation of polyethylene. Polym. Degrad. Stab. 18, 73-87. https://doi.org/10.1016/0141-3910(87)90084-X (1987).

11. Rajandas, H., Parimannan, S., Sathasivam, K., Ravichandran, M. \& Yin, L. S. A novel FTIR-ATR spectroscopy based technique for the estimation of low-density polyethylene biodegradation. Polym. Test. 31, 1094-1099 (2012).

12. Albertsson, A.-C., Barenstedt, C., Karlsson, S. \& Lindberg, T. Degradation product pattern and morphology changes as means to differentiate abiotically and biotically aged degradable polyethylene. Polymer 36, 3075-3083 (1995).

13. Koutny, M., Amato, P., Muchova, M., Ruzicka, J. \& Delort, A.-M. Soil bacterial strains able to grow on the surface of oxidized polyethylene film containing prooxidant additives. Int. Biodeterior. Biodegrad. 63, 354-357 (2006).

14. Fontanella, S. et al. Comparison of the biodegradability of various polyethylene films containing pro-oxidant additives. Polym. Degrad. Stab. 95, 1011-1021 (2010).

15. Gravoiul, K. et al. Transcriptomics and lipidomics of the environmental strain Rhodococcus ruber point out consumption pathways and potential metabolic bottlenecks for polyethylene degradation. Environ. Sci. Technol. 51, 5172-5181. https://doi.org/10.1021/ acs.est.7b00846 (2017).

16. Pathak, V. M. \& Navneet. Review on the current status of polymer degradation: A microbial approach. Bioresour. Bioprocess. 4, 15. https://doi.org/10.1186/s40643-017-0145-9 (2017).

17. Grover, A., Gupta, A., Chandra, S., Kumari, A. \& Khurana, S. M. P. Polythene and environment. Int. J. Environ. Sci. https://doi. org/10.6088/ijes.2014050100103 (2015).

18. Cappelletti, M. et al. Phenotype microarray analysis may unravel genetic determinants of the stress response by Rhodococcus aetherivorans BCP1 and Rhodococcus opacus R7. Res. Microbiol. 167, 766-773. https://doi.org/10.1016/j.resmic.2016.06.008 (2016).

19. Zampolli, J., Zeaiter, Z., Di Canito, A. \& Di Gennaro, P. Genome analysis and -omics approaches provide new insights into the biodegradation potential of Rhodococcus. Appl. Microbiol. Biotechnol. 103, 1069-1080. https://doi.org/10.1007/s00253-018-9539-7 (2018).

20. Yoon, M. G., Jeon, H. J. \& Kim, M. N. Biodegradation of polyethylene by a soil bacterium and alkB cloned recombinant cell. J. Bioremed. Biodegrad. 3, 4. https://doi.org/10.4172/2155-6199.1000145 (2012).

21. Jeon, H. J. \& Kim, M. N. Functional analysis of alkane hydroxylase system derived from Pseudomonas aeruginosa E7 for low molecular weight polyethylene. Int. Biodeterior. Biodegrad. 103, 141-146 (2015).

22. Santo, M., Weitsman, R. \& Sivan, A. The role of the copper-binding enzyme-laccase-in the biodegradation of polyethylene by the actinomycete Rhodococcus ruber. Int. Biodeterior. Biodegrad. 84, 204-210 (2013).

23. Di Gennaro, P., Rescalli, E., Galli, E., Sello, G. \& Bestetti, G. Characterization of Rhodococcus opacus R7, a strain able to degrade naphthalene and $o$-xylene isolated from a polycyclic aromatic hydrocarbon-contaminated soil. Res. Microbiol. 152, 641-651 (2001).

24. Orro, A. et al. Genome and phenotype microarray analyses of Rhodococcus sp. BCP1 and Rhodococcus opacus R7: Genetic determinants and metabolic abilities with environmental relevance. PLoS One 10, e0139467. https://doi.org/10.1371/journal.pone.01394 67 (2015).

25. Maniatis, T., Fritsch, E. F. \& Sambrook, J. Molecular Cloning: A Laboratory Manual (Cold Spring Harbor Laboratory, 1982).

26. Krueger, F. Trim Galore: A wrapper tool around Cutadapt and FastQC to consistently apply quality and adapter trimming to FastQ files. https://www.bioinformatics.babraham.ac.uk/projects/trim_galore/ (2015).

27. Manconi, A. et al. Removing duplicate reads using graphics processing units. BMC Bioinform. 17(Suppl 12), 346. https://doi.org/ 10.1186/s12859-016-1192-5 (2016).

28. Di Gennaro, P. et al. Genome sequence of Rhodococcus opacus strain R7, a biodegrader of mono- and polycyclic aromatic hydrocarbons. Genome Announc. 2, 4. https://doi.org/10.1128/genomeA.00827-14 (2014).

29. Kim, D. et al. TopHat2: Accurate alignment of transcriptomes in the presence of insertions, deletions and gene fusions. Genome Biol. 14, R36. https://doi.org/10.1186/gb-2013-14-4-r36 (2013).

30. Trapnell, C. et al. Transcript assembly and quantification by RNA-Seq reveals unannotated transcripts and isoform switching during cell differentiation. Nat. Biotechnol. 28, 511-515. https://doi.org/10.1038/nbt.1621 (2010).

31. Trapnell, C. et al. Differential analysis of gene regulation at transcript resolution with RNA-seq. Nat. Biotechnol. 31, 46-53. https:// doi.org/10.1038/nbt.2450 (2013). 
32. Delcher, A. L., Bratke, K. A., Powers, E. C. \& Salzberg, S. L. Identifying bacterial genes and endosymbiont DNA with Glimmer. Bioinformatics 23, 673-679. https://doi.org/10.1093/bioinformatics/btm009 (2007).

33. Aziz, R. K. et al. The RAST server: Rapid annotations using subsystems technology. BMC Genomics 9, 75. https://doi.org/10.1186/ 1471-2164-9-75 (2008).

34. Altschul, S. F., Gish, W., Miller, W., Myers, E. W. \& Lipman, D. J. Basic local alignment search tool. J. Mol. Biol. 215, 403-410. https://doi.org/10.1016/S0022-2836(05)80360-2 (1990).

35. Thompson, J. D., Higgins, D. G. \& Gibson, T. J. CLUSTAL W: Improving the sensitivity of progressive multiple sequence alignment through sequence weighting, position-specific gap penalties and weight matrix choice. Nucleic Acids Res. 22, 4673-4680. https:// doi.org/10.1093/nar/22.22.4673 (1994).

36. The UniProt Consortium. UniProt: A worldwide hub of protein knowledge. Nucleic Acids Res. 47(D1), D506-D515. https://doi. org/10.1093/nar/gky1049 (2019).

37. Kanehisa, M., Furumichi, M., Tanabe, M., Sato, Y. \& Morishima, K. KEGG: New perspectives on genomes, pathways, diseases and drugs. Nucleic Acids Res. 45(D1), D353-D361. https://doi.org/10.1093/nar/gkw1092 (2017).

38. Mi, H. et al. PANTHER version 14: More genomes, a new PANTHER GO-slim and improvements in enrichment analysis tools. Nucleic Acids Res. 47(D1), D419-D426. https://doi.org/10.1093/nar/gky1038 (2019).

39. Bustin, S. A. The MIQE Guidelines: Minimum Information for Publication of Quantitative Real-Time PCR Experiments. Clin. Chem. 55, 611-622 (2009).

40. Su, X., Guo, L., Ding, L., Qu, K. \& Shen, C. Induction of viable but nonculturable state in Rhodococcus and transcriptome analysis using RNA-seq. PLoS One 11, e0147593. https://doi.org/10.1371/journal.pone.0147593 (2016).

41. Tian, W., Arakaki, A. K. \& Skolnick, J. EFICAz: A comprehensive approach for accurate genome-scale enzyme function inference. Nucleic Acids Res. 32, 6226-6239 (2004).

42. Sigrist, C. J. A. et al. PROSITE: A documented database using patterns and profiles as motif descriptors. Brief. Bioinform. 3, 265-274 (2002).

43. Berman, H. M. et al. The protein data bank. Nucleic Acids Res. 28, 235-242 (2000).

44. Rose, R. W., Bruser, T., Kissinger, J. C. \& Pohlschroder, M. Adaptation of protein secretion to extremely high-salt conditions by extensive use of the twin-arginine translocation pathway. Mol. Microbiol. 45, 943-950 (2002).

45. Bendtsen, J. D., Nielsen, H., Widdick, D., Palmer, T. \& Brunak, S. Prediction of twin-arginine signal peptides. BMC Bioinform. 6, 167 (2005).

46. Armenteros, J. J. A. et al. SignalP 5.0 improves signal peptide predictions using deep neural networks. Nat. Biotechnol. 37, 420-423. https://doi.org/10.1038/s41587-019-0036-z (2019).

47. Bradford, M. M. A rapid and sensitive method for the quantitation of microgram quantities of protein utilizing the principle of protein-dye binding. Anal. Biochem. 72, 248-254. https://doi.org/10.1016/0003-2697(76)90527-3 (1976).

48. Zampolli, J. et al. Transcriptomic analysis of Rhodococcus opacus R7 grown on o-xylene by RNA-Seq. Front. Microbiol. 11, 1808. https://doi.org/10.3389/fmicb.2020.01808 (2020).

49. Reiss, R. et al. Laccase versus laccase-like multi-copper oxidase: A comparative study of similar enzymes with diverse substrate spectra. PLoS One 8, e65633. https://doi.org/10.1371/journal.pone.0065633 (2013).

50. Mogharabia, M. \& Faramarzia, M. A. Laccase and laccase-mediated systems in the synthesis of organic compounds. Adv. Synth. Catal. 356, 897-927 (2014).

51. Goosens, V. J., Monteferrante, C. G. \& van Dijl, J. M. The Tat system of Gram-positive bacteria. Biochim. Biophys. Acta Mol. Cell Res. 1843, 1698-1706 (2014).

52. Laczi, K. et al. Metabolic responses of Rhodococcus erythropolis PR4 grown on diesel oil and various hydrocarbons. Appl. Microbiol. Biotechnol. 99, 9745-9759 (2015).

53. de Montellano, P. R. O. et al. Potential drug targets in the Mycobacterium tuberculosis cytochrome P450 system. J. Inorg. Biochem. 180, 235-245 (2018).

54. Rosłoniec, K. Z. et al. Cytochrome P450 125 (CYP125) catalyses C26-hydroxylation to initiate sterol side-chain degradation in Rhodococcus jostii RHA1. Mol. Microbiol. 74, 1031-1043 (2009).

55. Zampolli, J., Collina, E., Lasagni, M. \& Di Gennaro, P. Biodegradation of variable-chain-length $n$-alkanes in Rhodococcus opacus R7 and the involvement of an alkane hydroxylase system in the metabolism. AMB Express 4, 73. https://doi.org/10.1186/s13568014-0073-4 (2014).

56. Ami, D. et al. Pathological ATX3 expression induces cell perturbations in E. coli as revealed by biochemical and biophysical investigations. Int. J. Mol. Sci. 22, 943. https://doi.org/10.3390/ijms22020943 (2021).

57. Mantsch, H. H. \& McElhaney, R. N. Phospholipid phase transitions in model and biological membranes as studied by infrared spectroscopy. Chem. Phys. Lipids 57, 213-226 (1991).

\section{Acknowledgements}

We would like to thank our student Daniele Vezzini for support in the experimental work.

\section{Author contributions}

J.Z. and P.D.G. conceived and designed the research and the manuscript. J.Z. performed the experiments and wrote the draft manuscript; D.A. performed FTIR analyses and together with A.N. analyzed FTIR statistic data and wrote the part on the manuscript relative to FTIR. A.O. and A.M. performed the RNA-seq data analysis. All authors provided critical feedback and contributed to the final manuscript.

\section{Funding}

This work was funded by 2020-ATE-0460 from University of Milano-Bicocca.

\section{Competing interests}

The authors declare no competing interests.

Additional information

Supplementary Information The online version contains supplementary material available at https://doi.org/ 10.1038/s41598-021-00525-x.

Correspondence and requests for materials should be addressed to P.D.G.

Reprints and permissions information is available at www.nature.com/reprints. 
Publisher's note Springer Nature remains neutral with regard to jurisdictional claims in published maps and institutional affiliations.

(c) (i) Open Access This article is licensed under a Creative Commons Attribution 4.0 International License, which permits use, sharing, adaptation, distribution and reproduction in any medium or format, as long as you give appropriate credit to the original author(s) and the source, provide a link to the Creative Commons licence, and indicate if changes were made. The images or other third party material in this article are included in the article's Creative Commons licence, unless indicated otherwise in a credit line to the material. If material is not included in the article's Creative Commons licence and your intended use is not permitted by statutory regulation or exceeds the permitted use, you will need to obtain permission directly from the copyright holder. To view a copy of this licence, visit http://creativecommons.org/licenses/by/4.0/.

(C) The Author(s) 2021 